\title{
A novel technique for detecting the misbehavior nodes in wireless adhoc networks
}

\author{
Kumar Narayanan ${ }^{1 *}$, R. Anandan², Swaraj Paul Chinnaraju ${ }^{3}$, A. Manikandan ${ }^{4}$ \\ ${ }^{1}$ Department of Computer Science \& Engineering, Vels Institute of Science, Technology \& Advanced Studies(VISTAS), Chennai, India. \\ ${ }^{2}$ Department of Computer Science \& Engineering, Vels Institute of Science, Technology \& Advanced Studies(VISTAS), Chennai, India. \\ ${ }^{3}$ Department of Computer Science \& Engineering, Vels Institute of Science, Technology \& Advanced Studies(VISTAS), Chennai, India. \\ ${ }^{4}$ Department of Computer Science \& Engineering, Vels Institute of Science, Technology \& Advanced Studies(VISTAS), Chennai, India. \\ *Corresponding author E-mail:dr.kumarnarayanan@gmail.com
}

\begin{abstract}
Wireless adhoc network acts as the most emerging platform in the current trend that plays a vital role in the field of sensing, analyzing the network route, rectifying the problem within the network. This also pays route for many advanced studies. Observing aid depletion attacks on the routing layers is the main objective of this paper. It also attempts to entirely disable community nodes by draining their battery strength immediately. Such an attempt to drain their battery strength is said to be as depletion attack, and these attacks are not specific to any particular protocol. Under the worst case, even a single depletion can empower a community-wide usage by $\mathrm{O}(\mathrm{N})$, where $\mathrm{N}$ is the total number of network nodes used.
\end{abstract}

Keywords: Wireless Ad-hoc sensor network, depletion attack.

\section{Introduction}

\section{Purpose}

The primary goal of this research is to construct routing protocols by means of Parno, Luk, Gaustad, and Perrig (PLGP), which is comfortable, loss of protection from these depletion attacks, because they drain the life from community nodes.

\section{Paper scope}

We explore aid depletion assaults on the routing protocol layer, which permanently disable networks by using fast draining nodes battery electricity. These Depletion attacks aren't specific to any precise protocol, but as an alternative depend on routing protocols. At the extreme worst end, only a single depletion can push forward more network power usage at the rate of $\mathrm{O}(\mathrm{N})$, wherein $\mathrm{N}$ inside the quantity of network nodes. These methods mitigate these forms of attacks, such as a brand new evidence-of-concept protocol that provably bounds the harm due to Depletions throughout the packet forwarding section.

\section{Performance analysis}

Packet delivery ratio. The proportional ratio between the range of packets originated through the software layer CBR sources and the wide variety of packets obtained through the CBR sink at the final destination.

Routing price. The proportional ratio among the overall number of bytes of packets with information transmitted up to the duration of simulation and the overall bytes of packets retrieved by means of the CBR sink at the receiving end.

Packet overhead. The number of transmitted routing packets; as an instance, a HELLO or TC message despatched over four hops might be counted as 4 packets in this metric.
Mean latency. It is the average time taken from the time of transferring the first packet of information to the time taken for receiving the same first packet at the receiver's end.

Routing Matrices. The earliest metric proposed for locating the most to be had bandwidth route is ETX. The ETX metric of every link 1 is defined as ETXl $1 \mathrm{Pl}$, in which pl denotes the packet loss opportunity on link 1 at the MAC layer. Pl is envisioned by means of proactively broadcasting the dedicated hyperlink Probe packets. These Depletion assaults are not unique to any unique protocol, but alternatively rely upon the houses of many popular lessons of routing protocols. In the worst case, a single depletion can grow network-wide energy utilization via a issue of $\mathrm{O}(\mathrm{N})$, in which $\mathrm{N}$ in the variety of community nodes. Its outline strategies to mitigate those sorts of assaults, along with a brand new evidence-ofconcept protocol that provably bounds the damage as a result of Depletion s throughout the packet forwarding phase.

Depletion assault occurs inside the network, any node in the network that when infected and if its behavior changing and thus the whole network behavior changes, then this form of nodes are known as Malicious node. If when malicious nodes survives in the community power that have been using through each and every nodes will increases drastically. The malicious node has been area inside the network uniquely. 


\section{System analysis}

\section{Existing system}

In supply routing protocols, It indicates the way to specify the route through the community that are too longer than the premier, and this needs to be specified by the malicious packet source, and wasting strength at nodes that are in between the source and he destination, that can forward the packets. An adversary comprises packets with purposely added routing loops.

\section{Disadvantages}
1. Power outages
2. Environmental disasters
3. Low efficiency
4. Various DOS attacks
5. Secure level is low

\section{Proposed system}

In the proposed system, It shows that a clean-sheet relaxed routing protocol for the sensor network via Parno, Luk, Gaustad, and Perrig (PLGP), and it can be modified to prove its withstand depletion assaults throughout the section where packet forwarding are done. The unique model of the protocol, despite the fact that designed for safety, is prone to depletion assaults. PLGP comprises of a network structure discovery segment, followed by using a packet forwarding segment, with the previous optionally repeated on a hard and fast agenda to make certain that topology information remains modern.

In this phase, They display that a easy-slate comfy sensor community routing protocol by using Parno et al. ("PLGP" from here on) may be changed to prove its withstand Depletion attacks for the duration of the packet forwarding section. The authentic model of the protocol, even though designed for protection, is liable to Depletion assaults.

\section{Advantages}

1. Protect from the depletion attacks

2. Secure level is high

3. Boost up the Battery power.

\section{Security against depletion attacks}

Here, its regulate the forwarding phase of PLGP to provably keep away from the above-cited assaults. First we introduce the nobacktracking assets, satisfied for a given packet if and best if it continuously makes development toward its vacation spot within the logical network deal with space

\section{Detection and mitigation of depletion attacks}

As the paper has now sincerely illustrated the way wherein Depletion assaults broaden in a network and its devastating effects at the community. We at the moment are in role to now design a mechanism or approach by which we are able to limit this impact such attack. In a WSN network while dispatching the message packets from the transmitter node to receiving node, it gets forwarded through the intermediate nodes that are available inside the community and this flow will be kept till it reaches the receiving node. In the proposed machine we overcome the Depletion assault with the aid of performing various tests for its correctness that guarantees that packets would not go into limitless loop thus resulting in the drainage of battery and crashing the entire community. For validation we define function Secure packet forward (p).

The existence of single node stretch attacker on a communitynode that comprises of around 30 nodes after removing the route from the source and its duration limits, causes a greater effect. Harmful and suspicious nodes are measured in phrases of the precipitated range of the greatest path, in number of hops.

Network hyperlinks become saturated at 10,000 messages in line with 2 nd (even under the absence of the stretch assault), however the adversary can gain the same results with the aid of forwarding an order of importance lesser messages at a stretch attack.

\section{Modules list}

There are three modules.

-Network Topology

-Energy efficient model

- Simulation

\section{Module description}

Network topology

\section{Stretch attack}

In this module, Every node has to send hey message to all the other nodes which allows finding the details of transmission. Once if a node finds hey message from another node (neighbor), it continues a touch report to save records about the neighbor. The neighbor nodes are found for all the nodes in that network using multicast socket. The Cluster Head is elected primarily based on Range, Battery and Mobility.

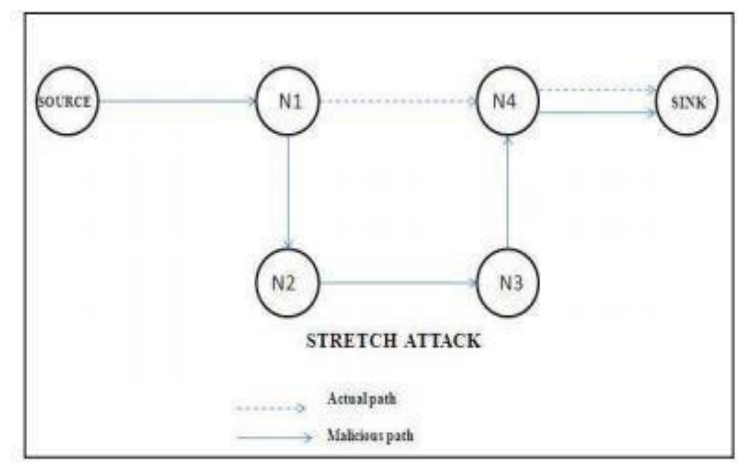

Fig. 3.1: Stretch attack

Node initialization is carried out whilst thinking about the various problems of transferring packets through the identified route from a source node_o' to a destination node_d' in a wireless advert-hoc network. Source node transmits the information packet to vacation spot node via intermediate nodes. If there malicious node present within the network, it travels long distance. The chance node takes the most variety of route to reach the sink node.

Adversary constructs artificially lengthy routes traversing each node inside the community. It causes packets to traverse larger than most advantageous quantity of nodes that doesn't lie on topquality course to process packets. Theoretical restriction, electricity usage increase of aspect $\mathrm{O}(\min (\mathrm{N}, \lambda))$, wherein $\mathrm{N}$ is the quantity of nodes within the network and $\lambda$ is the maximum course duration allowed.

And then probably much less unfavorable consistent with packet than the carousel assault, because the no of hops per packet is bounded through the range of network nodes.

Carousel Attack:

In this module, The paths are generated dynamically relying on the number of created nodes in network. The supply node transmits the statistics packets via the random route, which can be handed via more than one node. Adversary sends packets with routes composed of a chain of loops. 


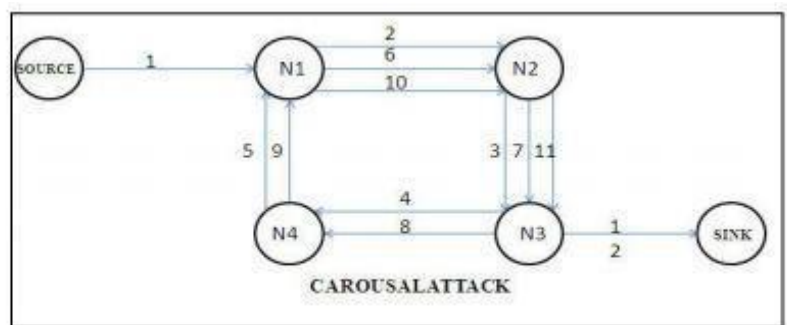

Fig. 3.2: Arousel attack

It exploits constrained verification of message headers at forwarding nodes. Malicious node growth the path duration beyond number of nodes in network. Theoretical restrict: power utilization increase by way of a component of $\mathrm{O}(\lambda)$, wherein $\lambda$ is the maximum course length.

\section{Energy efficient version}

We expect that using estimation method, the distance between every node in the MANET to its neighbor nodes will be determined. The transmission energy of each node plays a vital role in finding the connectivity of the community. Each node can interchange its transmission electricity stage during the transmission time. A node can use a exclusive electricity level for each multicast tree in which it participates. All the nodes use single-directional receiver we put in force the electricity efficient genetic algorithm in Manet.

\section{Three simulation}

Evaluate the effect of the Virtual transmissions technique offered. We measured the overall performance of HEF whilst the quantity of mother and father is various from one to three. The outcomes are acquired from based at the above topology.

\section{System design}

\section{Network topology}

Every node sends messages to permit other nodes to discover it. Once a node detects messages from every other node (neighbor), it creates and manages a contact document to store data approximately the neighbor. Using multicast socket all nodes are used to come across the neighbor nodes. This Model fits many utility that collect records from surroundings as consumer targeted fees.

\section{ITA version}

We propose an ITA spine deployment algorithm. The set of rules has four steps to be followed: right from the starting stage deployment to decide the starting location of $\mathrm{k}$ BNs.

1. RN affiliation, which greedily assigns the $\mathrm{RNs}$ to associate with okay BNs based totally on the contemporary round of BNs deployment to limit the common backbone community get admission to put off cost.

2. Adaptation of the positions of ok BNs primarily based at the association of $\mathrm{n}$ Rns.

3. Checking the connectivity to make sure that the $\mathrm{k}$ BNs forms a related spine network.

4. The set of rules runs iteratively via the steps 2,3 , and until both the goal function cannot be advanced any greater or the BN community will become disconnected.

5. Evaluate the effect of the Virtual transmissions technique provided. We measured the overall performance of this approach whilst the quantity of parents is various from one to 3 . The results are received from based totally on the above topology

\section{Result and discussions}

\section{Module 1: home page module}

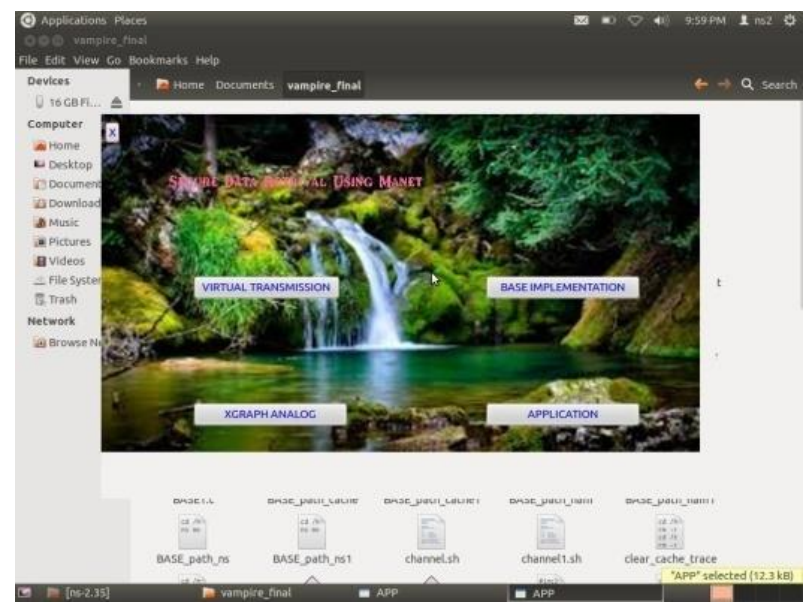

Home page module shown in this page

\section{Module 2: NS2 configuration}
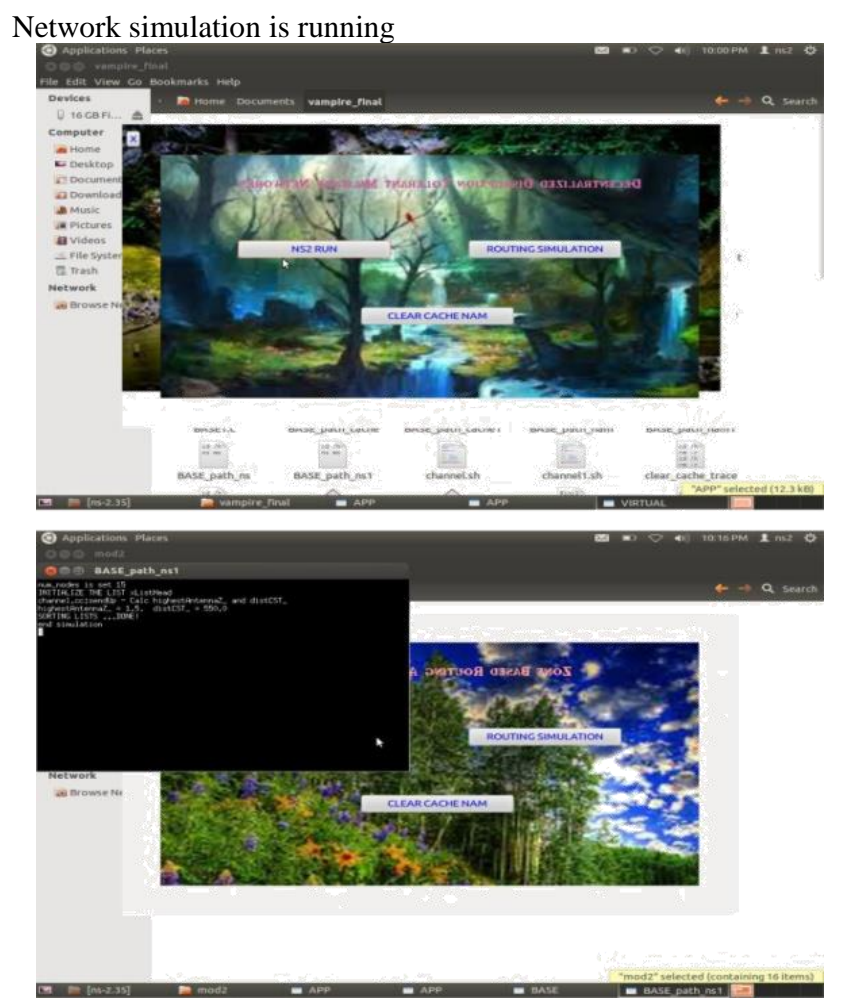

Module 3: node initializations 


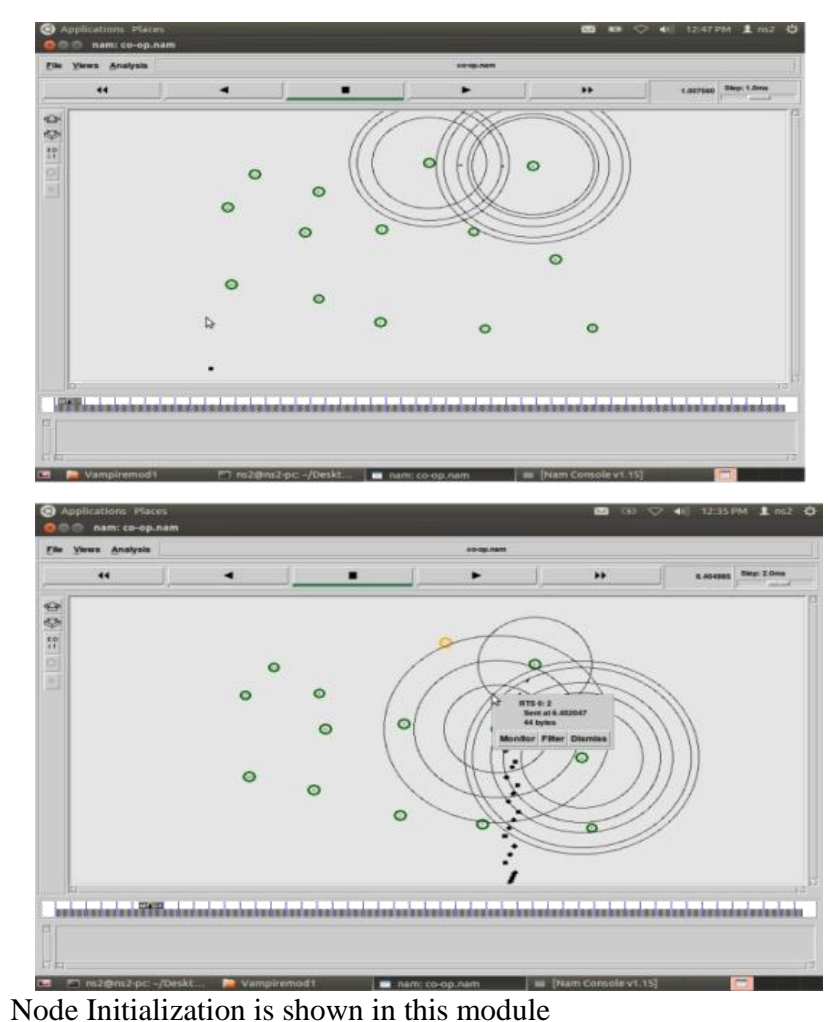

Node Initialization is shown in this module

\section{Module 4: node formations}

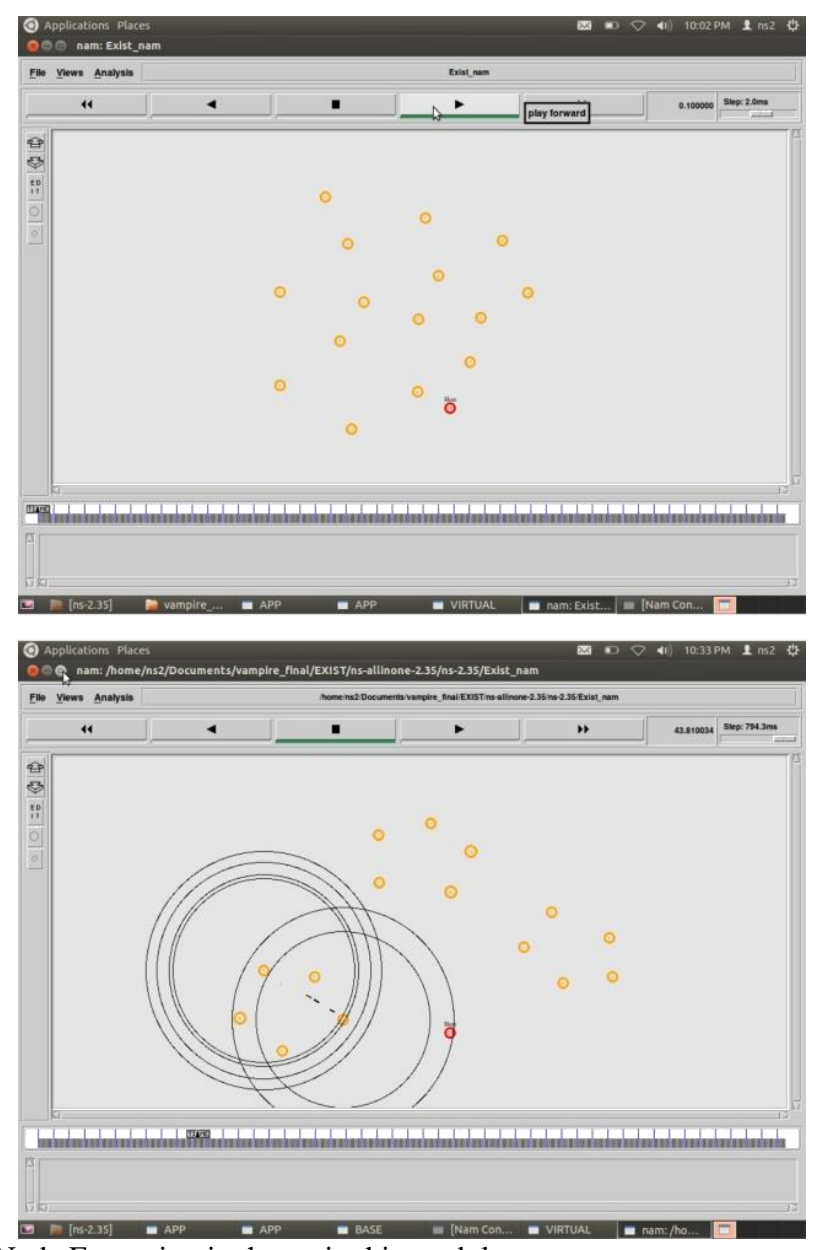

Node Formation is shown in this module

Module 5: sensor 1 identify the node 0 and 12 on moving

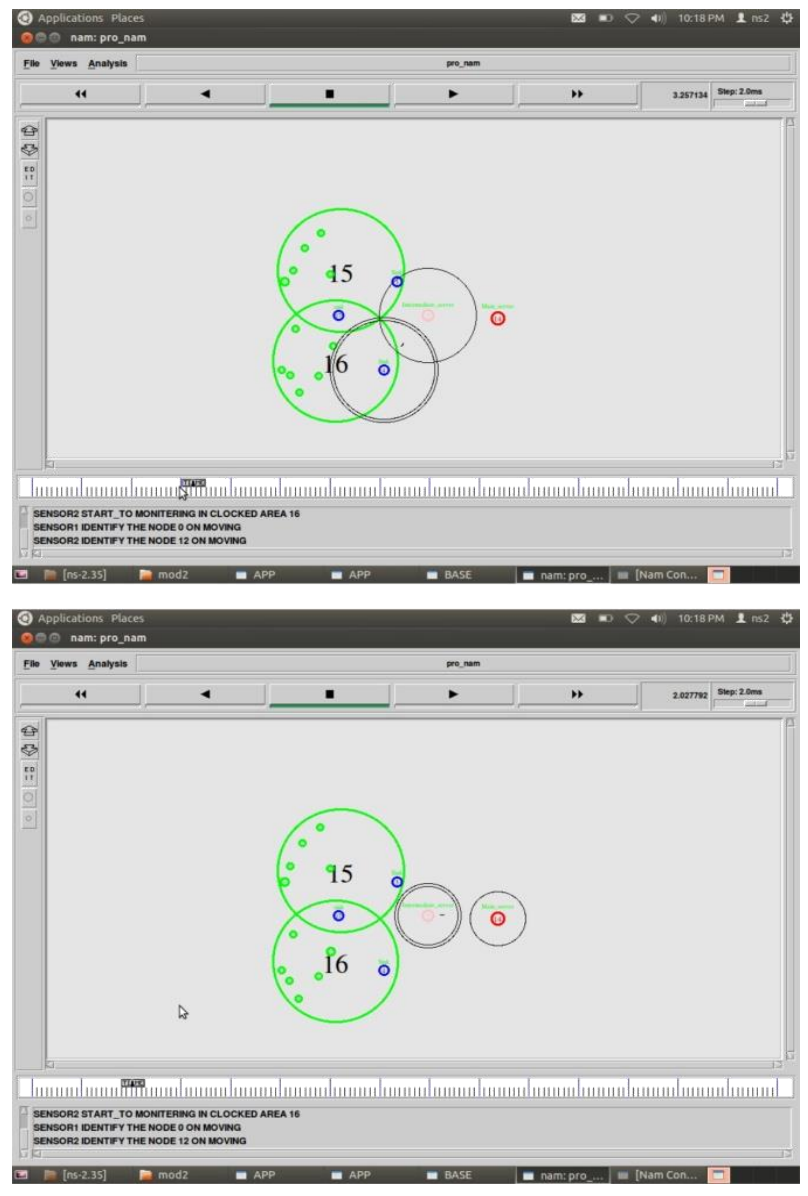

Module 6: sensor 2 identify the node 0 and 12 on moving

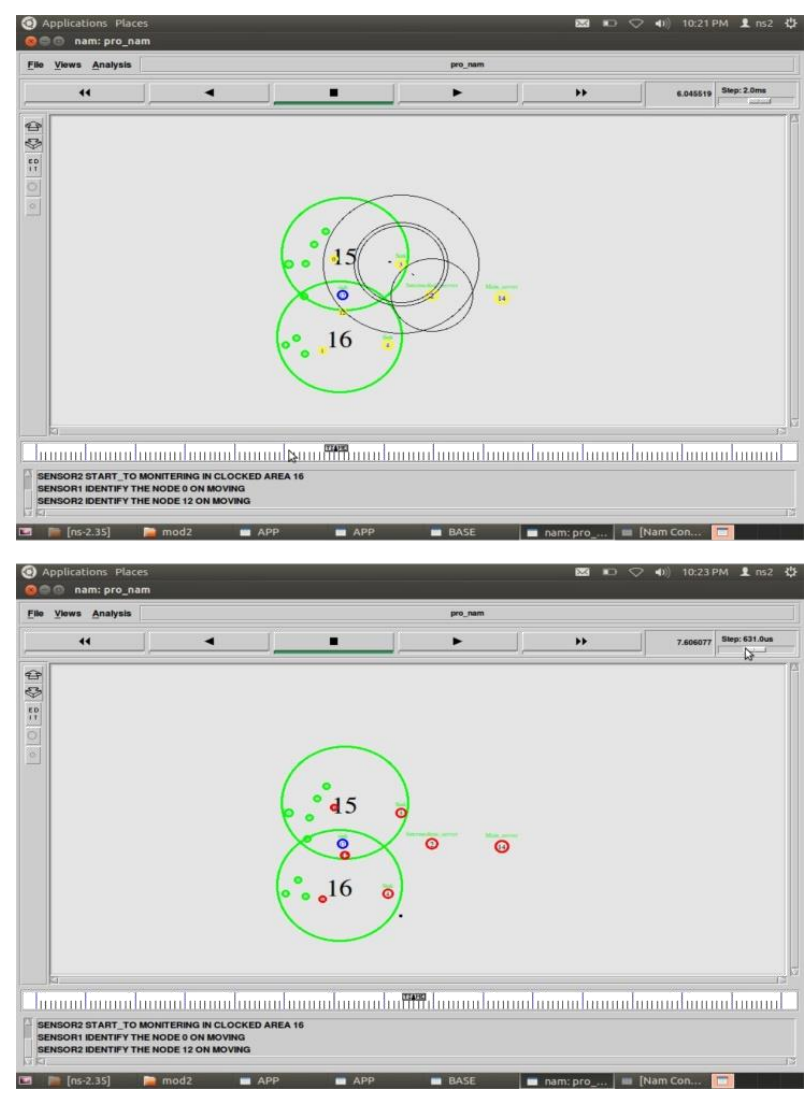

\section{A.3 Graph}

Delay 


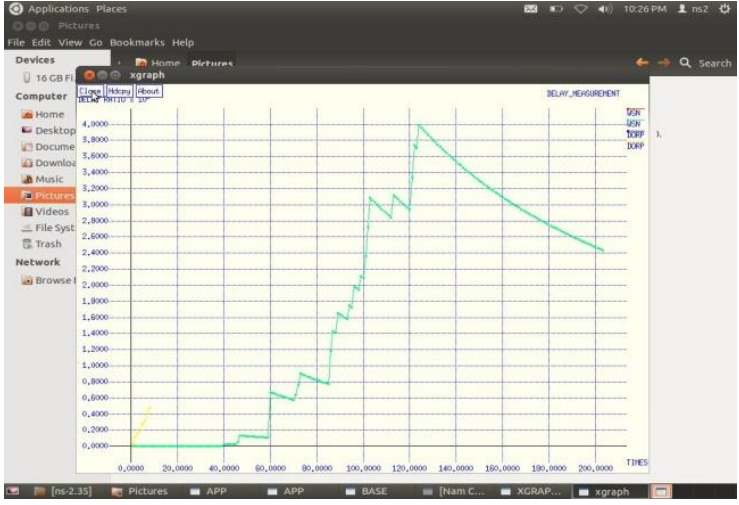

\section{Throughput workload}

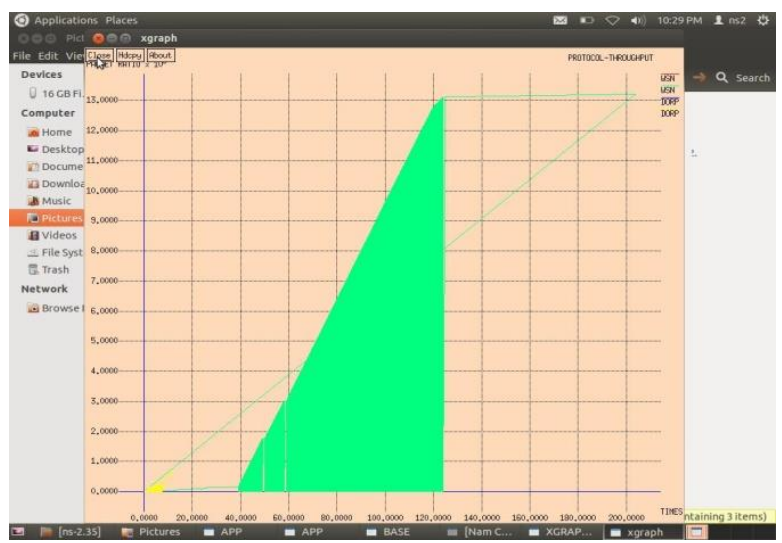

\section{Channel measurement}

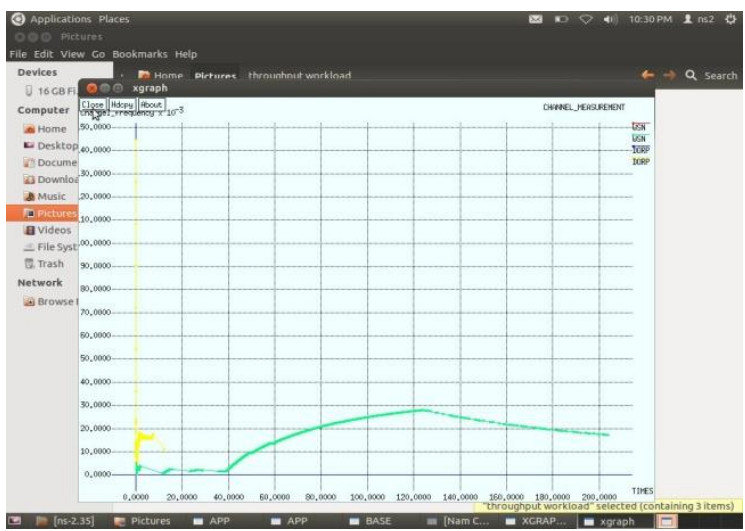

\section{Total loss}

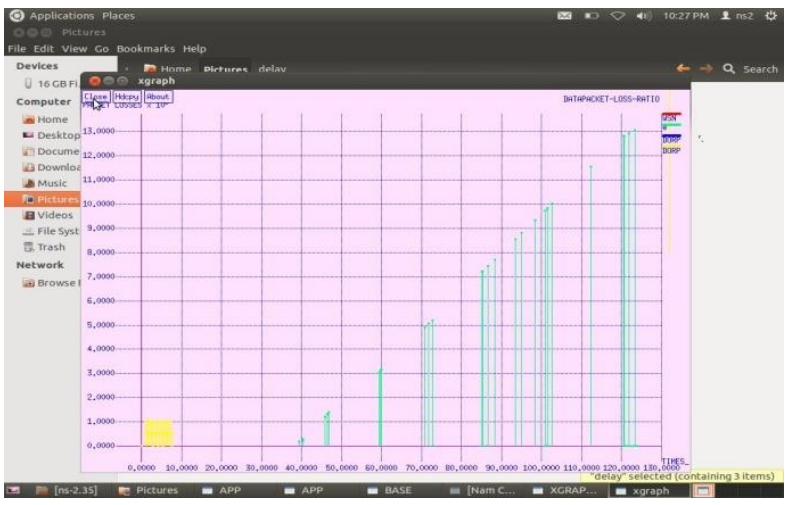

\section{Conclusion}

In this paper, cope with the properties of routing protocol attacks within the wi-fi advert hoc networks In order to conquer the depletion or malicious attacks in WSN, the facts transmission is carried within the depended on path of the networks. Our proposed technique addresses the depletion attacks in the wi-fi ad hoc networks when in comparison to the prevailing methods. In the depletion assault, the two styles of assaults may arisen the complete networks into fall apart, overall electricity intake level will increase, and allocates lengthy routing path and so on. And the 2 styles of attacks are: In the Carousel assault, attackers introduce some packet inside a course tranquil in the form of continuous loops, so that the equal node seems within the direction of communication commonly and the other assault is stretch assault also focused on resource steering, attackers construct falsely distant routes, doubtlessly travelling to every node inside the network. And additionally stretch attack, will increase packet lane duration, thus leading the packets to be executed by using a number of nodes this is self-governing of hop rely down the directly route stuck among the challenger and packet target. This assault increases the routing length and postpone very lots inside the networks and also inadequate with the aid of the quantity of allowable entries within the resource route. In this paper we also proposed new method to locate the misbehavior nodes in the wireless advert hoc networks. The results of this experiments had confirmed that our proposed novel technique works efficaciously whilst as compared to previous methods.

\section{References}

[1] Rajesh Khanna M, Divya S \& Rengarajan A, "Securing Wireless Ad-Hoc Sensor Network", International Journal of Innovative Research in Computer and communication Engineering(IJIRCC), Vol.2, (2014).

[2] Sivakumar K \& Murugapriya P, "Efficient Detection and Elimination of Depletion attacks in Wireless Ad-Hoc Sensor Networks", IJIRCCE, Vol.2, (2014).

[3] Mohana M \& Kaviya P, "A Survey on Secure Packet Transmission against Depletion Attack in Wireless Ad-hoc Sensor Networks", IJARCCE, Vol.3, No.11, (2014).

[4] Vijayanand G \& Muralidharan R, 'Overcome Depletion attacks Problem In Wireless Ad-Hoc Sensor Network By Using Distance Vector Protocols", IJCSMA, Vol.2, No.1, (2014).

[5] Anand J \& Sivachandar K , 'Depletion Attack Detection in Wireless Sensor Network", IJESIT, Vol.3, No.4, (2014).

[6] Vasserman EY \& Hopper N, "Depletion attacks: Draining life from wireless ad-hoc sensor networks", IEEE Transactions on Mobile Computing, Vol.12, No.2, (2013).

[7] Khandakar A, "Step by Step Procedural Comparison of DSR, AODV and DSDV Routing protocol", 4th International Conference on Computer Engineering and Technology (ICCET), Vol.40, (2012).

[8] Ning X \& Cassandras CG, "On Maximum Lifetime Routing in Wireless Sensor Networks", Joint 48th IEEE Conference on Decision and Control and 28th Chinese Control Conference, (2009).

[9] Acs G, Buttyan L \& Vajda I, "Provably Secure On-Demand Source Routing in Mobile Ad Hoc Networks", IEEE Trans. Mobile Computing, Vol.5, No.11, (2006), pp.1533-1546.

[10] Deng J, Han R \& Mishra S, "Defending against Path based DoS Attacks in Wireless Sensor Networks", Alexandria, Virginia, USA, (2005). 